\title{
COVID -19 AND DIABETES MELLITUS
}

\section{Medicine \\ Dr Gopal Chandra Nath*}

Consultant

Consultant Medicine, Department of Medicine, Guwahati Neurological Research Centre and Hospital, Guwahati, $781006,{ }^{*}$ Corresponding Author

\begin{abstract}
Introduction: Covid 19 is an infectious disease caused by severe acute respiratory syndrome coronavirus 2 (SARS-COV-2). Diabetes Mellitus is a significant predictor of mortality and morbidity in patients with Covid 19. Aims and objective: This study was done to understand the Covid 19 disease in diabetes and its management. Materials and methods: In this study we tried to understand the protocols of management of covid 19 in patients with Diabetes mellitus. Results: What we found in our patients is that rigorous glucose monitoring with careful consideration of drug interactions might decrease the worsening of symtoms and the complications. Conclusion: As patients with Diabetes Mellitus are having severe disease in term of Covid 19 infection, these patients should be strictly monitored and early isolation, diagnosis and early management might collectively contribute to the better control of the disease and outcome. Due to limited number of cases studied, more such studies are needed to understand the pathophysiology of the association between Diabetes and Covid 19.
\end{abstract}

\section{KEYWORDS}

Diabetes Mellitus, Covid 19, Management.

\section{INTRODUCTION:}

Covid 19 is an infectious disease caused by severe acute respiratory syndrome coronavirus 2 (SARS-COV-2).It was first identified in december 2019 in Wuhan, China and now it is causing a Pandemic. The clinical spectrum of Covid 19 is heterogenous. Symtoms vary from mild flu like illness to severe acute respiratory distress syndrome, multiorgan failure, shock, cardiac injury and death. ${ }^{1}$ RT-PCR is the standard diagnostic test for Covid 19 as it detects the nucleic acid of SARS-CoV-2 in throat swabs. It has been observed that most severe cases of Covid 19 have occurred in elderly and in patients with underlying comorbidities such as Diabetes Mellitus, chronic lung and renal disease and cancer, although the exact mechanisms are still not understood. ${ }^{2}$ Diabetes Mellitus is a complex heterogeneous group of metabolic conditions characterized by increased levels of blood glucose due to impaired insulin action and/or insulin secretion. ${ }^{3}$ Chronic inflammation, impairment of immune response by Covid 19, increased coagulation activity might be among the underlying mechanisms of the association between the two disease. ${ }^{4}$ The negative impact of diabetes on weakening individuals immune system illustrates the link between the disease and being infected with COVID 19. Diabetes Mellitus is a significant predictor of mortality and morbidity in patients with Covid 19.It is largely unknown how exactly the immune response occurs in these patients or if the virus itself interfere with insulin sccretion. In patients with Diabetes there is increased synthesis of inflammatory cytokines, Advanced glycation end products that mediate inflammation. This inflammatory response may be the reason of worst outcomes in Diabetes Mellitus. ${ }^{6}$ This study was done to understand the Covid 19 disease in diabetes and its management.

\section{MATERIALS AND METHODS:}

Various studies are done throughout the world to understand the pathogenegis of Covid 19 and its impact on people with various comorbidities. In this study we tried to understand the protocols of management of Covid 19 in patients with Diabetes mellitus. We admitted 30 patients of Covid 19 in our hospital till date. Out of which 05 patients had diabetes. All of the Diabetic patients were admitted in Intensive care unit and had severe disease.

\section{RESULTS:}

There is no data available regarding the appropriate management of patients with diabetes mellitus infected with COVID -19. What we found in our patients is that rigorous glucose monitoring with careful consideration of drug interactions might decrease the worsening of symtoms and the complications. A multidisciplinary team approach is needed to combact the complications. As diabetes has its own complications such as cardiovascular disease, nephropathy etc ,these patients should be given special attention since they are at higher risk for severe infection with Covid 19 and death. Although hyperglycemia is usually the main concern but hypoglycaemic episodes should be given equal importance as a result of interplay between drug treatment, viral pathogenesis and the metabolic disturbances of Diabetes Mellitus. Drugs such as hydroxychloroquine can precipitate hypoglycaemic events in Diabetes. Therefore a dose adjustment of oral anti-diabetic drugs and insulin is necessary in order to prevent potential hypoglycaemic events.

\section{DISCUSSION:}

One Chinese meta-analysis showed that the most prevalent cardiovascular metabolic comorbities with Covid 19 were hypertension followed by Diabetes. In this report, patients with Diabetes had a two fold increase in risk of severe disease or require intensive care unit admission. ${ }^{2}$ Some studies did not find a clear association between diabetes and severe disease ${ }^{4}$ but we urged that patients with Diabetes Mellitus are prone to more severe disease, hence these patients should be strictly isolated so they are not infected or if infected, they should be strightly monitored so that the complications can be decreased.

\section{CONCLUSION:}

The present study highlights the relation between Covid 19 and Diabetes mellitus. As patients with Diabetes Mellitus are having severe disease in term of covid 19 infection, these patients should be strictly monitored and early isolation, diagnosis and early management might collectively contribute to the better control of the disease and outcome. Due to limited number of cases studied, more such studies are needed to understand the pathophysiology of the association between Diabetes and Covid -19 so that we can formulate appropriate drugs and decrease the complications and mortality in patients with Diabetes Mellitus.

\section{REFERENCES:}

1. Diabetes Canada: https://www.diabetes.ca/resources/tools---resources/faq-aboutDiabetes Canada: htt
covid-19-and-diabetes

2. Chen Y, Yang D,Cheng B et al.Clinical characteristics and outcomes of patients with diabetes and COVID-19 in association with glucose-lowering medication.Diabetes Care. 2020; 43: 1399-1407

3. American Diabetes Association. Diagnosis and classification of diabetes mellitus. Diabetes Care. 2014;37 Suppl 1:S81-S90. [PubMed] [Google Scholar]

Guo W, Li M,Dong Y et al. Diabetes is a risk factor for the progression and prognosis of COVID-19. Diabetes Metab Res Rev. 2020; (https//:doi.org.10.1002/dmrr.3319 published online March 31.)

published online March 31.)

5.
6. Yang JK, Feng Y, Yuan MY, et al. Plasma glucose levels and diabetes are independent predictors for mortality and morbidity in patients with SARS. Diabet Med. 2006;23:623-8. 